\title{
The effect of walking speed on the foot inter-segment kinematics, ground reaction forces and lower limb joint moments
}

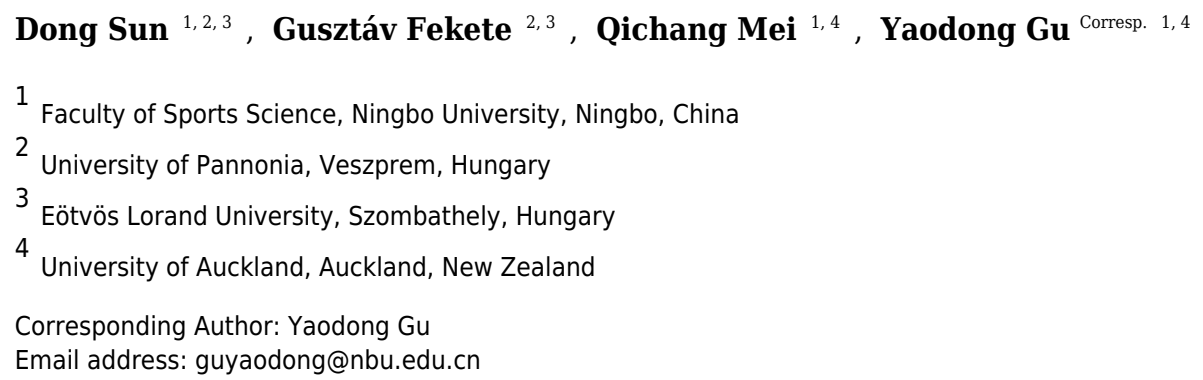

Background: Normative foot kinematic and kinetic data with different walking speed will benefit rehabilitation programs and improving gait performance. The purpose of this study was to analyze foot kinematics and kinetics differences between slow walking (SW), normal walking (NW) and fast walking (FW) of healthy subjects. Methods: Ten healthy male subjects participated in this study, who were asked to carry out walk at a self-selected speed. After measuring and averaging the results of normal walking, the subjects were asked to perform $25 \%$ slower and $25 \%$ faster walk respectively. Temporal-spatial parameters, kinematics of the tibia (TB), hindfoot (HF), forefoot (FF) and hallux (HX) and ground reaction forces (GRFs) were recorded while the subjects walked at averaged speeds of $1.01 \mathrm{~m} / \mathrm{s}$ (SW), $1.34 \mathrm{~m} / \mathrm{s}$ (NW) and $1.68 \mathrm{~m} / \mathrm{s}$ (FW). Results: Hindfoot relative to tibia (HF/TB) and forefoot relative to hindfoot (FF/HF) dorsiflexion increased in FW, while hallux relative to forefoot (HX/FF) dorsiflexion decreased. Increased peak eversion and peak external rotation in HF/TB were observed in FW with decreased peak supination in FF/HF. GRFs were increased significantly with walking speed. The peak values of the knee and ankle moments in the sagittal and frontal planes significantly increased during FW compared with SW and NW. Discussion: Limited HF/TB and FF/HF motion of SW was likely compensated for increased HX/FF dorsiflexion. Although small angle variation in HF/TB eversion and FF/HF supination during FW may have profound effects for foot kinetics. Higher HF/TB external rotation contributed to the forefoot push-off the ground while the center of mass progresses forward in FW, therefore accompanied by higher FF/HF abduction in FW. Increased peak vertical GRF in FW may affected by decreased stance duration time, the biomechanical mechanism maybe the change in vertical center of mass (COM) height and increase leg stiffness. Walking speed changes accompanied with modulated sagittal plane ankle moments to alter the braking GRF during loading response. 
The findings of foot kinematics, ground reaction forces, and lower limb joint moments among healthy males may set a reference to distinguish abnormal and pathological gait patterns. 
1 The effect of walking speed on the foot inter-segment kinematics, ground reaction forces and

2 lower limb joint moments

3

4 Dong Sun ${ }^{1,2,3}$, Gusztáv Fekete ${ }^{2,3}$, Qichang Mei ${ }^{1,4}$, Yaodong Gu ${ }^{1,4}$

$5 \quad{ }^{1}$ Faculty of Sports Science, Ningbo University, Ningbo, Zhejiang Province, China.

$6 \quad{ }^{2}$ Faculty of Engineering, University of Pannonia, Veszprem, Hungary.

$7 \quad{ }^{3}$ Savaria Institute of Technology, Eötvös Loránd University, Szombathely, Vas State, Hungary.

$8{ }^{4}$ Auckland Bioengineering Institute, University of Auckland, Auckland, New Zealand.

9

10 Corresponding author:

11 Yaodong $\mathrm{Gu}^{1,4}$

12 No. 616, Fenghua Road, Jingbei District, Ningbo, 315211, China

E-mail Address: guyaodong@hotmail.com 


\section{ABSTRACT}

Background: Normative foot kinematic and kinetic data with different walking speed will benefit rehabilitation programs and improving gait performance. The purpose of this study was to analyze foot kinematics and kinetics differences between slow walking (SW), normal walking (NW) and fast walking (FW) of healthy subjects. Methods: Ten healthy male subjects participated in this study, who were asked to carry out walk at a self-selected speed. After measuring and averaging the results of normal walking, the subjects were asked to perform $25 \%$ slower and $25 \%$ faster walk respectively. Temporal-spatial parameters, kinematics of the tibia (TB), hindfoot (HF), forefoot (FF) and hallux (HX) and ground reaction forces (GRFs) were recorded while the subjects walked at averaged speeds of $1.01 \mathrm{~m} / \mathrm{s}(\mathrm{SW}), 1.34 \mathrm{~m} / \mathrm{s}(\mathrm{NW})$ and $1.68 \mathrm{~m} / \mathrm{s}(\mathrm{FW})$. Results: Hindfoot relative to tibia $(\mathrm{HF} / \mathrm{TB})$ and forefoot relative to hindfoot $(\mathrm{FF} / \mathrm{HF})$ dorsiflexion increased in $\mathrm{FW}$, while hallux relative to forefoot $(\mathrm{HX} / \mathrm{FF})$ dorsiflexion decreased. Increased peak eversion and peak external rotation in $\mathrm{HF} / \mathrm{TB}$ were observed in $\mathrm{FW}$ with decreased peak supination in $\mathrm{FF} / \mathrm{HF}$. GRFs were increased significantly with walking speed. The peak values of the knee and ankle moments in the sagittal and frontal planes significantly increased during FW compared with SW and NW. Discussion: Limited HF/TB and FF/HF motion of SW was likely compensated for increased $\mathrm{HX} / \mathrm{FF}$ dorsiflexion. Although small angle variation in $\mathrm{HF} / \mathrm{TB}$ eversion and $\mathrm{FF} / \mathrm{HF}$ supination during FW may have profound effects for foot kinetics. Higher HF/TB external rotation contributed to the forefoot push-off the ground while the center of mass progresses forward in FW, therefore accompanied by higher FF/HF abduction in FW. Increased peak vertical GRF in FW may affected by decreased stance duration time, the biomechanical mechanism maybe the change in vertical center of mass (COM) height and increase leg stiffness. Walking speed changes accompanied with modulated sagittal plane ankle moments to alter the braking GRF during loading response. The findings of foot kinematics, ground reaction forces, and lower limb joint moments among healthy males may set a reference to distinguish abnormal and pathological gait patterns. 


\section{INTRODUCTION}

Walking speed is a parameter that is interconnected with the measures of mobility and daily activities. It is also worth mentioning that the ability of changing walking speed is a personal measure of adaptation with regard to the lower limbs which can be quantified by various gait parameters. Significant relevance have been found between walking speed and spatial-temporal parameters, lower limb kinematics, kinetics and electromyography activities in both healthy and pathology adults (Andriacchi, Ogle and Galante, 1977; Chiu and Wang, 2007; Chung and Wang, 2010; Dubbeldam et al., 2010; Holden, Chou and Stanhope, 1997; Tulchin et al., 2009; Van Hoeve et al., 2017; Fukuchi, Fukuchi and Duarte, 2018).

It has been also demonstrated that an initial increase in stride length is followed by increased cadence together with increased walking speed in healthy adults (Kirtley, 2006). Changing walking speed has been found to affect sagittal plane kinematics as well. Increased peak knee flexion and ankle dorsiflexion angles during stance phase with higher walking velocity was reported (Umberger and Martin, 2007). While other authors proved that significant differences were found at the hindfoot and forefoot sagittal kinematics in healthy adults with different walking speeds (Tulchin et al., 2009). Dubbeldam and colleagues (2010) found that walking speed significantly affected foot and ankle kinematics, which showed minimum tibio-talar plantarflexion and maximum hallux dorsiflexion decreased during toe-off phase as walking speed decreasing. Grant and Chester (2015) found all significant changes in the magnitude of foot angles during variable walking speed only occurred in the sagittal plane. Non-sagittal foot kinematics of adult subjects were also reported in recent studies. However, with increase in walking speed, the hindfoot was found to be more inverted on frontal plane and more adducted on transverse plane with respect to the tibia during late stance (Caravaggi, Leardini and Crompton, 2010). Gait kinetic parameters showed a strong correlation with increase in walking speed, particularly the ground reaction forces (GRFs) and joint moments. It was reported that vertical and horizontal GRFs were significantly affected by gait speed, especially during the loading response phase and mid-stance phase (White et al., 1996). A linear correlation between peak propulsive force and joint moments 
82

83

84

and walking speed was reported (Lelas et al., 2003). The addition of intra-foot kinetics to multisegment foot modeling may provide additional insight into foot and ankle function under different walking speed. A three-segment kinetic foot model was mentioned in a previous study, which incorporating kinetic parameters and calculating joint moments and powers of ankle, mid-tarsal, and 1st metatarsophalangeal (MTP) joints (Bruening, Cooney and Buczek, 2012). While Dixon et al., (Dixon, Böhm and Döderlein, 2012) attempt to use a four segments Oxford foot model (OFM) to calculate ankle and midfoot kinetics and compare ankle kinetics with one foot segment Plug-ingait (PIG) model, the masses of the forefoot and hindfoot were set to half the value of the whole foot. Overestimate of ankle power when using one-segment foot model was main suggested in their study.

The multi-segment foot models have been extensively used by dividing foot into multiple rigid segments over the past decades (Caravaggi et al., 2010; MacWilliams, Cowley and Nicholson, 2003). The OFM has demonstrated robust validity and repeatability with an overall foot intersegment angles throughout a gait cycle in both adults and children (Carson et al., 2001). Numerous studies have examined the effects of gait speed on temporal-spatial parameters, joint kinematics, ground reaction forces and muscle activities (Chiu and Wang, 2007; Goldberg and Stanhope, 2013; Kang and Dingwell, 2008). However the effect of walking speed remained controversial on foot kinematics. A validated reference set, with regard to the inter-segmental kinematics of normal foot, could be a substantial result in order to compare abnormal or pathological gait to clinical gait. Therefore, the main objective of this study was to investigate difference in foot inter-segment kinematics, GRFs and lower limb joint moments variables between self-selected walking speed $(\mathrm{NW})$, relatively slower walking speed (SW) and relatively faster walking speed (FW) in matured healthy subjects. We hypothesized that with increasing walking speed, foot inter-segment kinematics and range of motion (ROM) would significantly increase in the sagittal, frontal and transverse planes. For kinetic variables, increased GRFs and joint moments were also hypothesized with increasing walking speed. 
109

110

111

112

113

114

115

116

117

118

119

120

121

122

123

124

125

126

127

\section{METHODS}

\section{Subjects}

Ten healthy male subjects (age: $24.6 \pm 2.3$ years, height: $1.72 \pm 0.3 \mathrm{~m}$, weight: $67.6 \pm 8.3 \mathrm{~kg}$, BMI: $22.85 \pm 1.22 \mathrm{~kg} / \mathrm{m}^{2}$ ) without misalignment of lower limbs and any orthopedic surgeries history of lower extremities volunteered to participate in this study. All the subjects were rear-foot strikers and the dominant feet are the right one based on the test of kicking a soccer ball, so all statistics concerning the tests are based on data from the right feet. Informed consent were obtained before taking part in the experiments. Ethics approval was obtained from Ethics committee of Ningbo University (No. RAGH20170516).

\section{Experimental protocol and procedure}

The three-dimensional marker trajectories were captured using a VICON MX motion analysis system (Oxford Metrics Ltd, Oxford, UK), with 8 cameras and sampling at $200 \mathrm{~Hz}$. Reflective markers were placed according to the marker set of a modified version of the Oxford Foot Model (Stebbins et al., 2006). The OFM is a multi-segment foot model used to calculate intersegment foot kinematics. Simplified complex foot structure to three rigid segments (tibia, hindfoot, and forefoot) and one vector (hallux). A set of 36 markers $(9 \mathrm{~mm})$ were placed bilaterally on bony landmarks to model the tibia, hindfoot, forefoot and hallux. Static marker placement on the right foot was shown in Figure 1. All inter-segment motions except hallux motion were threedimensional. The kinematic sign conventions used for OFM inter-segment angles were shown in Table 1. The OFM have been shown to provide consistent angular motion throughout the gait cycle for adults and children. However, marker placement variations may have potential influence on joint angles assessment. For each subject, the right foot were measured in three sequential walking speed sessions between which the markers were not removed. In order to prevent high variability between subjects, only one experienced laboratory technician were asked to attach the markers to all participants. Calibration of the system was performed before the gait trials. GRFs data were collected using a $600 \times 400 \mathrm{~mm}$ force platform (AMTI, Watertown, MA, USA) at a sampling frequency of $1000 \mathrm{~Hz}$. The marker and force data were synchronized. 
136

137

138

139

140

141

142

143

144

145

146

147

148

149

150

151

152

153

154

155

156

157

158

159

160

161

162

Initially, the subjects were required to walk at their own comfortable walking speed (normal walking, NW). The walking speed was recorded using Brower timing lights (Brower Timing System, Draper, UT, USA), positioned $1.2 \mathrm{~m}$ apart, centering the force plate. In the second and third session the subjects were asked to walk 25\% slower than NW (slow walking, SW) and 25\% faster than NW (fast walking, FW) of their comfortable walking speed (NW) respectively. During each session, 8 successful trials were obtained for each walking speed with less than $5 \%$ variance and within $5 \%$ of the predefined walking speed.

\section{Insert Figure 1: OFM marker placement. The three markers (RD1M, RMMA, and RPCA) were} removed after static modeling.

\section{Insert Table 1: Kinematic sign conventions used for OFM inter-segment angles}

\section{Data analyses}

Temporal-spatial parameters, including walking speed, stride length, stride time, stance duration and cadence were calculated and recorded. All gait data were labelled and processed in VICON Nexus software (Version 1.8.5, VICON). Euler angles were calculated for inter-segment rotation following the sequence of Grood and Suntay (flexion, adduction, and rotation) (Grood and Suntay, 1983). The following three dimensional motion of angles were measured: hindfoot relative to tibia (HF/TB), forefoot relative to hindfoot $(\mathrm{FF} / \mathrm{HF})$, forefoot relative to tibia $(\mathrm{FF} / \mathrm{TB})$ and hallux relative to forefoot $(\mathrm{HX} / \mathrm{FF})$ (sagittal plane only). Kinematic and kinetic traces were visually checked and inconsistent trials removed. Eight successful measurements were recorded when subjects landed with right foot on the force platform. For each trial, gait events were detected by the use of the vertical GRF to determine initial heel contact and toe-off with a threshold of $30 \mathrm{~N}$. OFM parameters such as the angular motion at heel contact (HC), toe-off (TO), peak values and range of motion (ROM) in the stance phase were derived with regard to the different walking speeds. Peak propulsive, braking and vertical GRFs were also recorded and analyzed. Vertical 
163

164

165

166

167

168

169

170

171

172

173

174

175

176

177

178

179

180

181

182

183

184

185

186

187

188

189

average loading rate (VALR) was computed as first peak vertical loading GRF/time (from contact). The peak braking GRFs and peak propulsive GRFs were the peak negative and positive anterior-posterior GRFs.

OFM does not calculate moments, it is a kinematics-only model (Paterson et al., 2017). Therefore, the knee and ankle external moment data in the sagittal and frontal planes in this study were calculated using a standard full inverse dynamic model based on the VICON PIG model (Keenan et al., 2011). GRFs and joint moments were normalized and reported in body weight (BW) and Nm/kg. The foot kinematic and GRF data were filtered with a fourth-order zero-phase lag Butterworth filter having a cutoff frequency of $8 \mathrm{~Hz}$ and $20 \mathrm{~Hz}$, respectively. One gait cycle was defined as ipsilateral heel contact ground twice. Gait cycles were normalized in the complete range of motion in order to clearly distinguish major and minor variations in the patterns of the individual trials. Spatiotemporal parameters, foot kinematics, GRFs and joint moments were determined in case of each participant during the three different walking speeds. The collected foot inter-segment kinematics for analysis include heel contact, toe-off and peak angle values in a stance phase and angle curves during a gait cycle. The ROM was defined as the maximum minus minimum angle during stance phase.

\section{Statistical analyses}

The statistical analyses were performed in SPSS version 22.0 (SPSS Science, Chicago, Illinois, USA). All data were normally distributed which assessed by the Shapiro-Wilk test and are presented as mean \pm SD (Standard Deviation). One-way analysis of variance (ANOVA) was performed, followed by a post-hoc Bonferroni test, were used to test for significant differences in temporal-spatial parameters, foot inter-segment kinematics, GRFs and lower limb joint moments with changes of walking speed. Statistical significance was determined at the level of $p<0.05$.

\section{RESULTS}

Kinematics

\section{Temporal-spatial parameters}


Mean and standard deviation values of the temporal-spatial variables of SW, NW and FW were given in Table 2. The one-way ANOVA test proved that the temporal variables are statistically significant with regard to the three walking speeds. With increasing walking speed, the stride length and the cadence increased while the stance duration, stance phase (\% gait cycle) and stride time decreased.

Insert Table 2: Descriptive statistics of the temporal-spatial parameters (mean \pm SD) during three walking speeds.

\section{Hindfoot relative to tibia $(\mathrm{HF} / \mathrm{TB})$ motion}

Average foot inter-segment kinematics curves during gait cycle are shown in Figure 2. Selected crucial points and statistics data about foot motions during stance phase, values at heel contact (HC), toe-off (TO), maximum (max) and minimum (min) foot angles, ROM are included in Table 3. No significant differences were observed in the HF/TB motion between SW and NW in sagittal, frontal or transverse plane. Nevertheless, one-way ANOVA showed statistically significant difference between the movements. In sagittal plane, FW showed significantly higher heel contact dorsiflexion, peak dorsiflexion and dorsiflexion/plantarflexion (DF/PF) ROM. An increase in walking speed were corresponded with a significant increase of the peak values of $\mathrm{HF} / \mathrm{TB}$ dorsiflexion. In frontal plane, a significant increase in eversion (EV) at heel contact was found at higher walking speed during stance phase, while peak inversion angle was found lower in FW inversely. In transverse plane, significantly greater peak external rotation (ER) and ROM were found in FW.

\section{Forefoot relative to hindfoot (FF/HF) motion}

In the sagittal plane, peak FF/HF DF angle in $\mathrm{FW}$ was significantly greater than SW. Peak PF angle was found significantly higher in NW. Concerning ROM values, both SW and NW showed significantly higher DF/PF ROM compared with FW. In the frontal plane, there was a significantly larger supination in SW and NW at heel contact. In the transverse plane, adduction angles at IC 
217 and adduction/abduction (ADD/ABD) ROM were found significantly higher in SW. However,

218 maximum ABD angle was found significantly greater in FW.

219 Forefoot relative to tibia (FF/TB) motion

$220 \mathrm{FF} / \mathrm{TB}$ motion showed similar variations compared with HF/TB motion. In the sagittal plane,

221 FW showed more DF at IC and peak values compared with SW and NW. However, peak PF was

222 found significantly higher in NW. ROM (DF/PF) increased as walking speed escalated. No

223 significantly difference were found in peak SP and SP/PR ROM. In the transverse plane, ADD

224 angle at IC and maximum ADD angle showed significantly higher in SW and NW. Peak ABD and

$225 \mathrm{ADD} / \mathrm{ABD}$ ROM were also found significantly higher in FW.

226

227

228

229

230

231

232

233

234

235

236

237

238

239

240

241

242

243

\section{Hallux relative to forefoot $(\mathrm{HX} / \mathrm{FF})$ motion}

Only sagittal plane $\mathrm{HX} / \mathrm{FF}$ motion were concluded in this study, where the hallux was significantly less dorsiflexed in FW compared with SW and NW. Additionally, DF/PF ROM in SW and NW were significantly higher than FW.

\section{Insert Table 3: Foot kinematics with three walking speeds in the stance phase of gait.}

Insert Figure 2: Average foot kinematics $\left({ }^{\circ}\right)$ in sagittal, frontal and transverse planes during the gait cycle, shaded bands shows mean \pm standard deviation across three walking speeds. (SP, $S P$ ' and $S P$ ' represents the stance phase of $F W, N W$ and $S W$ conditions).

\section{Kinetics}

\section{Ground reaction forces (GRFs)}

GRFs of each subjects were normalized to body weight (BW). Normalized GRFs and VALR were shown in Table 4. In the anterior-posterior direction, peak braking force together with peak propulsive force was significantly higher in FW compared with SW, peak propulsive forces was significantly higher in FW compared to SW and NW. Peak vertical forces in FW condition was significantly higher than NW. VALR (BW/s) was found discriminatory effects between different 
244 walking speeds.

245 Lower limb joint moments

246 With regard to the parameters of joint moments, walking speed had a significant effect on knee 247 and ankle sagittal and frontal plane joint moments $(\mathrm{Nm} / \mathrm{kg})$. In the sagittal plane, peak knee flexion 248 moment in FW showed significantly higher compared with SW and NW. For peak knee extension 249 moment, FW showed significantly higher compared with SW and NW. Peak ankle dorsiflexion 250 moment in early stance phase showed significantly greater in FW compared with SW and NW. 251 Peak ankle plantarflexion moment in late stance phase was also significantly greater in FW

252

253

254

255

256

257

258

259

260

261

262

263

264

265

266

267

268

269

270

compared to SW and NW. In the frontal plane, statistically significant differences were also noted at the knee and ankle joints. Peak knee adduction and peak ankle eversion moments were significantly greater in FW compared to SW and NW. With an increasing walking speed, knee and ankle joint moments in sagittal and frontal planes increased as well, which indicates a positive, and possibly linear correlation between walking speeds and joint moments.

\section{Insert Table 4: Comparison of GRFs and joint moments at three walking speeds.}

\section{DISCUSSION}

The purpose of this study was to identify whether healthy adults have different foot intersegment kinematics, GRFs and lower limb joint moments when walking at a comfortable, relatively slower and faster walking speeds. This current study can provide valuable insight into how foot inter-segments interact with each other and how the GRFs and joint moments vary during different walking speed. The findings in one hand provide guidance what parameters may improve gait performance, while on the other hand it also gives a reference for clinical gait dysfunction diagnosis.

\section{Foot kinematics}

Significant difference has been revealed on the relative segmental foot motions ( $\mathrm{HF} / \mathrm{TB}, \mathrm{FF} / \mathrm{HF}$, $\mathrm{FF} / \mathrm{TB}$, and $\mathrm{HX} / \mathrm{FF}$ ) during $\mathrm{SW}, \mathrm{NW}$ and $\mathrm{FW}$. It must be mentioned as well, that the relative 
271 segmental foot motions observed in SW, NW and FW were nearly consistent with previous studies.

272 Our findings showed, that higher dorsiflexion angle was present at heel contact (HC) together with

273 higher maximum dorsiflexion angle and sagittal plane ROM during FW with regard to HF/TB.

274 These results are also consistent with the previous findings (Arnold et al., 2014; Grant and Chester,

275 2015; Jenkyn and Nicol, 2007). These studies also suggest that walking in a higher speed indicates

276 larger HF/TB DF angle. It was also concluded that in the heel contact HF/TB DF and peak HF/TB

277 DF angles increased with walking speed. The increase in HF/TB DF enables a larger stride length,

278 which could explain the temporal spatial results in this study. Greater peak EV and frontal plane

$279 \mathrm{ROM}$ of the HF/TB during stance phase of FW was also found. Previous studies provided evidence 280 that HF/TB peak EV could be used as an indicator for injuries risk due to excessive use (Chang et 281 al., 2014; Graham et al., 2011). More ER of HF/TB was also found in FW during mid-stance phase, 282 which is likely to be credited to the increased step length and cadence. These parameters could be 283 key factors to interpret higher HF/TB dorsiflexion and external rotation.

284 Maximum FF/HF DF angles increased significantly in higher walking speed, while the angle 285 variable was less than $3^{\circ}$. Furthermore, maximum FF/HF PF showed significantly greater in FW, 286 while the angle variable was less than $5^{\circ}$. This finding corresponds well with a previous study which also reported similar results in the FF/HF motion (Tulchin et al., 2009). Total sagittal FF/HF motion in plantar fasciitis subjects was found significantly greater compared to healthy subjects 289 (Chang et al., 2014). One advantage of using the OFM was the ability to assess frontal and transverse plane foot motion. In this study, decreased maximum FF/HF SP angle was observed in FW $\left(13.8^{\circ} \pm 0.5^{\circ}\right)$ compared to $\mathrm{SW}\left(14.6^{\circ} \pm 0.8^{\circ}\right)$ and $\mathrm{NW}\left(14.5^{\circ} \pm 0.6^{\circ}\right)$. Variation trend of $\mathrm{FF} / \mathrm{HF}$ $\mathrm{SP}$ angle at loading response was similar. Although differences in FF/HF motion between different walking speeds seem quite small at first glance, the stress and strain of the plantar fascia have shown a significant correlation with the intricate movements of the foot (Caravaggi et al., 2010). The plantar fascia tension would increase form $0.4 \mathrm{BW}$ to $0.7 \mathrm{BW}$ even when the arch angle changed $1^{\circ}$ during the first $50 \%$ of stance phase (Caravaggi, Leardini and Crompton, 2010). In the transverse plane, $\mathrm{FW}(+25 \% \mathrm{NW})$ showed more $\mathrm{FF} / \mathrm{HF} \mathrm{ABD}$ in mid-stance phase compared to 
298

299

300

301

302

303

304

305

306

307

308

309

310

311

312

313

314

315

316

317

318

319

320

321

322

323

324

SW $(-20 \% \mathrm{NW})$ and NW. From earlier studies it was deduced that differences were found in forefoot motion in the transverse plane between different walking speeds, $50 \% \mathrm{NW}, 75 \% \mathrm{NW}$ and NW, respectively (Dubbeldam et al., 2010). More FF/HF ABD in 50\% NW (-13.70 \pm 5.64$)$ was reported compared to $75 \% \mathrm{NW}\left(-12.85^{\circ} \pm 5.58\right)$ and $\mathrm{NW}\left(-12.44^{\circ} \pm 5.74\right)$. $\mathrm{FF} / \mathrm{HF}$ ABD angle showed similar variation in the speeds of $75 \% \mathrm{NW}$ and NW. However, higher FF/HF ABD angle was also found in FW (125\% NW) compared to 50\% NW. During mid-stance phase to toe-off phase, the ER of hindfoot contributed to the forefoot push-off over the ground while the center of mass (COM) progressed forward (Papi, Rowe and Pomeroy, 2015). For this reason, higher HF/TB ER angle may be accompanied by higher FF/HF ABD angle during walking with higher speed. The HX/FF was significantly more dorsiflexed in SW and NW throughout stance and additionally showed more ROM compared to FW. In our hypothesis, an increase in HX/FF DF angle was expected during walking with higher speed (FW), as a result of the higher loading, which would be transferred to the forefoot under higher walking speed. This is practically due to the roll-over mechanism of the foot (Samson et al., 2014). Nevertheless, lower HX/FF DF was observed during faster walking. A possible explanation is that the increased HX/FF DF angle may result in higher triceps surae muscle activity and more tension on the plantar fascia, which may restrain forefoot and hallux spreading in higher walking speed in this study (Neptune, Kautz and Zajac, 2001; Neptune, Zajac and Kautz, 2004). In our study, the foot inter-segment kinematic patterns between different walking speeds showed similar to that measured by Van Hoeve (Van Hoeve et al., 2017) using OFM foot model. While some decreased ROM (FF/HF sagittal plane and HX/FF sagittal plane) during stance phase were found in faster walking speed (FW) in this study. Caravaggi et al., (Caravaggi, Leardini and Crompton, 2010) found decreased range of motion in the sagittalplane between forefoot and hindfoot $(\mathrm{FF} / \mathrm{HF})$ with faster walking cadence. Which showed consistent FF/HF sagittal plane ROM between normal and faster walking speed. The decrease of FF/HF ROM was mainly caused by decreased maximal plantarflexion angle of forefoot. Tulchin et al., found maximal plantar flexion showed a $4.5^{\circ}$ change across walking speeds, as the duration of stance phase decreased. The decreased of HX/FF ROM during FW maybe a compensation 
325

326

327

328

329

330

331

332

333

334

335

336

337

338

339

340

341

342

343

344

345

346

347

348

349

350

351

mechanism for shorted stance time and decreasing FF/HF ROM.

In summary, in the sagittal plane, limited $\mathrm{HF} / \mathrm{TB}$ and $\mathrm{FF} / \mathrm{HF}$ motions of $\mathrm{SW}$ were likely compensated by the increased HX/FF DF in mid-stance phase. In the frontal plane, increased peak EV angle in HF/TB was observed during FW, which was also accompanied with a decreased peak $\mathrm{SP}$ angle in $\mathrm{FF} / \mathrm{HF}$. The angle variation is low in the frontal plane, which has profound effects on foot morphology and on foot kinetics (Ferber and Benson, 2011; Graham, Jawrani and Goel, 2011). In the transverse plane, higher HF/TB ER contributed to the forefoot push-off the ground, while the COM progressed forward, therefore it was accompanied by higher FF/HF ABD in FW (Wright et al., 2011). The foot motion differences between SW, NW and FW were consistent with the references which emphasizes the validity of the OFM for evaluating walking speed effects.

\section{Kinetics}

In this study, kinetic parameters show a consistent correlation with increases in walking speed. Increased vertical GRF, braking GRF (the posterior component of the GRF vector) and propulsive GRF (the anterior component of the GRF vector) were found in FW. The vertical GRF spikes at loading response to form the first impact peak, and then increases at mid-stance phase to approximately $100 \%$ to $110 \%$ body weight (BW) with a comfortable walking speed. Increased peak vertical GRF in FW was significantly affected by decreased stance duration time, which may minimize the change in vertical COM height, and in turn increase leg stiffness. Increased VALR was found during FW, peak vertical GRF and VALR have been reported as risk factors of barefoot walking. Zadpoor and Nikooyan (2006) found an earlier rise in vertical GRF and greater VALR during barefoot walking, which were believed as risk factors for tibia stress and plantar fasciitis. For normal adults walking, a change in the peak vertical GRF accompanied with a change in the horizontal component GRF (braking GRF and propulsive GRF). Braking and propulsive GRF increased with walking speeds from $1.0 \mathrm{~m} / \mathrm{s}-2.0 \mathrm{~m} / \mathrm{s}$, and propulsive GRF generated during pushoff phase could modulate walking speed (Nilsson and Thorstensson, 1989). Stride length and cadence could also influence propulsive GRF and increase with increasing walking speed, increases in peak propulsive GRF occur with increasing stride length at a higher walking speed 
352 (Martin and Marsh, 1992).

353 In the sagittal plane, peak knee and ankle joint moments during stance were found significantly 354 higher in FW compared to SW and NW. Lower limbs' peak joint moments were found systematically decreased with lower walking speed and could be well predicted based on walking speed among healthy individuals (Lelas et al., 2003). It was reported that peak sagittal plane moments had a predictive dependency on walking speed, and a correlation coefficient $(\mathrm{R}=0.86)$ of linear regression with the peak external knee flexion moment was found in stance phase (Schwartz, Rozumalski and Trost, 2008). Significant correlation between lower limb joint moment and spatial-temporal parameters was also reported in a previous. Individuals only experienced a significant increase in lower limb joint moments with an increased stride length or both stride length and cadence (Ardestani et al., 2016). In this study, both cadence and stride length were found with increased walking speed, therefore greater joint moments were found in FW. Walking speed changes accompanied with modulated sagittal ankle and knee moments to alter the braking GRF during loading response. Moreover, increased sagittal ankle moment is a consequence of increased walking speed. As peak GRFs and joint moments generating more mechanical strain on soft tissues during fast walking, the ability of the individuals to regulate the GRFs and joint moments is essential to soft tissue health (Dicharry, 2010). Furthermore, it has been shown that a knee joint flexor moment can be caused by a larger plantar flexor moment to the ankle joint (Simonsen et al., 1997). In the frontal plane, the knee adduction moment significantly increased in FW. Which was assessed as a marker of knee joint loading, the magnitude of knee adduction moment could reflect the medial compartment joint loading (Hunt et al., 2006). Further, due to the fact that lower limbs joints are interrelated and correlate by the kinematic and kinetic chain, alterations of foot kinematics may not only affect knee joint loads but may also have influence on the ankle joint (Graham et al., 2011). Kinetic parameters show a strong consistence with increases in walking speed, and was found mainly differences in FW compared to SW and NW.

Limitations

Several limitations of this current study should be considered. First of all, human walk with 
379

380

381

382

383

384

385

386

387

388

389

390

391

392

\section{3}

394

395

396

397

398

399

400

401

402

403

404

405

shoes in the real life, the foot kinematic and joint kinetic data of this study was only collected during barefoot condition. Thus the kinematic and kinetic patterns of shod conditions with different walking speed remains unknown. Secondly, the lowest walking speed selected in this study was about $1.0 \mathrm{~m} / \mathrm{s}$, which was considered not slow enough to represent or set reference for those patients with pathology on the foot, like diabetic foot or after stroke. Thirdly, intra-foot kinetics were not included in this study, the authors are willing to assess the effect of walking speed on foot inter-segment kinetics in the next study for additional insight into foot function during normal gait (Dixon, Böhm and Döderlein, 2012). Lastly, skin markers were used in this study as a non-invasive way to pursue segment movement and obtain kinematic data. However, skin markers oscillate and variability exists in identification of anatomical landmarks (Karlsson and Tranberg, 1999). To minimize the errors related to skin markers, a relatively small size markers $(9 \mathrm{~mm})$ compare to other protocols were used. Further, an experienced laboratory technician were asked to attach the markers to all participants to avoid inter-tester variability.

\section{CONCLUSIONS}

In summary, walking speed showed significant effect on foot inter-segment motions, ground reaction forces and joint moments in healthy subjects as hypothesized. In the sagittal plane, limited $\mathrm{HF} / \mathrm{TB}$ and $\mathrm{FF} / \mathrm{HF}$ motion of slow walking was likely to be compensated for increased HX/FF dorsiflexion during stance phase. In the frontal and transverse planes, increased HF/TB eversion and external rotation angles accompanied by decreased FF/HF supination in FW. Although the angle variation seems small, the subtle differences reflect complex motor control that adapts gaits in different walking speed. For kinetic parameters, increased peak vertical GRF in FW may affected by decreased stance duration time, the biomechanical mechanism under it may be smaller change in vertical center of mass (COM) height and increased leg stiffness under FW condition. Increased sagittal ankle moment is a consequence of increased walking speed. Walking speed changes accompanied with modulated sagittal plane ankle moments to alter the braking GRF during loading response. The propulsive GRF generated during push-off phase could modulate 
406

407

408

409

410

411

412

413

414

415

416

417

418

419

420

421

422

423

424

425

426

427

428

429

430

431

432

walking speed. Increased VALR and knee adduction moment in FW were assessed as marker of tibia injury risks and knee joint loading. Kinetic parameters show a strong consistence with increases in walking speed, and was found mainly differences in FW. The foot kinematic and kinetic data provided in this study from healthy subjects can be used as a reference set of normal gait parameters to distinguish abnormal gait patterns caused by different pathogenesis, and will provide basis for clinicians when deciding on treatment for abnormal and pathological gait. On the other hand, measuring foot inter-segment kinematics and kinetics related to walking speed could provide guidance for intervention strategies aimed at improving walking gait performance.

\section{REFERENCES}

Andriacchi TP., Ogle JA., Galante JO. 1977. Walking speed as a basis for normal and abnormal gait measurements. Journal of Biomechanics 10:261-268. DOI: 10.1016/00219290(77)90049-5.

Ardestani MM., Ferrigno C., Moazen M., Wimmer MA. 2016. From normal to fast walking: Impact of cadence and stride length on lower extremity joint moments. Gait and Posture 46:118-125. DOI: 10.1016/j.gaitpost.2016.02.005.

Arnold JB., Mackintosh S., Jones S., Thewlis D. 2014. Differences in foot kinematics between young and older adults during walking. Gait and Posture 39:689-694. DOI: 10.1016/j.gaitpost.2013.09.021.

Bruening DA., Cooney KM., Buczek FL. 2012. Analysis of a kinetic multi-segment foot model part II: Kinetics and clinical implications. Gait and Posture. DOI: 10.1016/j.gaitpost.2011.11.012.

Caravaggi P., Leardini A., Crompton R. 2010. Kinematic correlates of walking cadence in the foot. Journal of Biomechanics 43:2425-2433. DOI: 10.1016/j.jbiomech.2010.04.015.

Caravaggi P., Pataky T., Günther M., Savage R., Crompton R. 2010. Dynamics of longitudinal arch support in relation to walking speed: Contribution of the plantar aponeurosis. Journal of Anatomy 217:254-261. DOI: 10.1111/j.1469-7580.2010.01261.x. 
433 Carson MC., Harrington ME., Thompson N., O’Connor JJ., Theologis TN. 2001. Kinematic 434 analysis of a multi-segment foot model for research and clinical applications: A 435 repeatability analysis. Journal of Biomechanics 34:1299-1307. DOI: 10.1016/S0021$436 \quad 9290(01) 00101-4$.

437 Chang R., Rodrigues PA., Van Emmerik REA., Hamill J. 2014. Multi-segment foot kinematics 438 and ground reaction forces during gait of individuals with plantar fasciitis. Journal of 439 Biomechanics 47:2571-2577. DOI: 10.1016/j.jbiomech.2014.06.003.

440 Chiu MC., Wang MJ. 2007. The effect of gait speed and gender on perceived exertion, muscle 441 activity, joint motion of lower extremity, ground reaction force and heart rate during normal 442 walking. Gait and Posture 25:385-392. DOI: 10.1016/j.gaitpost.2006.05.008.

443 Chung MJ., Wang MJJ. 2010. The change of gait parameters during walking at different 444 percentage of preferred walking speed for healthy adults aged 20-60 years. Gait and 
460

461

462

463

464

465

466

467

468

469

470

471

472

473

474

475

476

477

478

479

480

481

482

483

484

485

486

46:1176-1183. DOI: 10.1016/j.jbiomech.2013.01.001.

Graham RB., Costigan PA., Sadler EM., Stevenson JM. 2011. Local dynamic stability of the lifting kinematic chain. Gait and Posture 34:561-563. DOI: 10.1016/j.gaitpost.2011.06.022.

Graham ME., Jawrani NT., Goel VK. 2011. Evaluating Plantar Fascia Strain in Hyperpronating Cadaveric Feet Following an Extra-osseous Talotarsal Stabilization Procedure. Journal of Foot and Ankle Surgery 50:682-686. DOI: 10.1053/j.jfas.2011.07.005.

Grant J., Chester V. 2015. The Effects of Walking Speed on Adult Multi-segment Foot Kinematics. Journal of Bioengineering \& Biomedical Science 05:181-183. DOI: 10.4172/2155-9538.1000156.

Grood ES., Suntay WJ. 1983. A Joint Coordinate System for the Clinical Description of ThreeDimensional Motions: Application to the Knee. Journal of Biomechanical Engineering. DOI: $10.1115 / 1.3138397$.

Van Hoeve S., Leenstra B., Willems P., Poeze M., Meijer K. 2017. The effect of age and speed on foot and ankle kinematics assessed using a 4-segment foot model. Medicine (United States). DOI: 10.1097/MD.0000000000007907.

Holden JP., Chou G., Stanhope SJ. 1997. Changes in knee joint function over a wide range of walking speeds. Clinical Biomechanics 12:375-382. DOI: 10.1016/S0268-0033(97)00020$\mathrm{X}$.

Hunt MA., Birmingham TB., Giffin JR., Jenkyn TR. 2006. Associations among knee adduction moment, frontal plane ground reaction force, and lever arm during walking in patients with knee osteoarthritis. Journal of Biomechanics 39:2213-2220. DOI: 10.1016/j.jbiomech.2005.07.002.

Jenkyn TR., Nicol AC. 2007. A multi-segment kinematic model of the foot with a novel definition of forefoot motion for use in clinical gait analysis during walking. Journal of Biomechanics 40:3271-3278. DOI: 10.1016/j.jbiomech.2007.04.008.

Kang HG., Dingwell JB. 2008. Effects of walking speed, strength and range of motion on gait stability in healthy older adults. Journal of Biomechanics 41:2899-2905. DOI: 
487

488

489

490

491

492

493

494

495

496

497

498

499

500

501

502

503

504

505

506

507

508

509

510

511

512

513

10.1016/j.jbiomech.2008.08.002.

Karlsson D., Tranberg R. 1999. On skin movement artefact-resonant frequencies of skin markers attached to the leg. Human Movement Science 18:627-635. DOI: 10.1016/S01679457(99)00025-1.

Keenan GS., Franz JR., Dicharry J., Croce U Della., Kerrigan DC. 2011. Lower limb joint kinetics in walking: The role of industry recommended footwear. Gait and Posture 33:350355. DOI: 10.1016/j.gaitpost.2010.09.019.

Kirtley C. 2006. Observational gait analysis. In: Clinical Gait Analysis :Theory And Practice. 267-278. DOI: 10.1016/B978-0-443-10009-3.50019-9.

Lelas JL., Merriman GJ., Riley PO., Kerrigan DC. 2003. Predicting peak kinematic and kinetic parameters from gait speed. Gait and Posture 17:106-112. DOI: 10.1016/S09666362(02)00060-7.

MacWilliams BA., Cowley M., Nicholson DE. 2003. Foot kinematics and kinetics during adolescent gait. Gait and Posture 17:214-224. DOI: 10.1016/S0966-6362(02)00103-0.

Martin PE., Marsh AP. 1992. Step length and frequency effects on ground reaction forces during walking. Journal of Biomechanics 25:1237-1239. DOI: 10.1016/0021-9290(92)90081-B.

Neptune RR., Kautz SA., Zajac FE. 2001. Contributions of the individual ankle plantar flexors to support, forward progression and swing initiation during walking. Journal of Biomechanics 34:1387-1398. DOI: 10.1016/S0021-9290(01)00105-1.

Neptune RR., Zajac FE., Kautz SA. 2004. Muscle force redistributes segmental power for body progression during walking. Gait and Posture 19:194-205. DOI: 10.1016/S09666362(03)00062-6.

NILSSON J., THORSTENSSON A. 1989. Ground reaction forces at different speeds of human walking and running. Acta Physiologica Scandinavica 136:217-227. DOI: 10.1111/j.17481716.1989.tb08655.x.

Papi E., Rowe PJ., Pomeroy VM. 2015. Analysis of gait within the uncontrolled manifold hypothesis: Stabilisation of the centre of mass during gait. Journal of Biomechanics 
48:324-331. DOI: 10.1016/j.jbiomech.2014.11.024.

515

516

517

518

519

520

521

522

523

524

525

526

527

528

529

530

531

532

533

534

535

536

537

538

539

540

Paterson KL., Hinman RS., Metcalf BR., Bennell KL., Wrigley T V. 2017. Plug-in-Gait calculation of the knee adduction moment in people with knee osteoarthritis during shod walking: Comparison of two different foot marker models. Journal of Foot and Ankle Research. DOI: 10.1186/s13047-017-0187-4.

Samson W., Van Hamme A., Sanchez S., Chèze L., Jan SVS., Feipel V. 2014. Foot roll-over evaluation based on 3D dynamic foot scan. Gait and Posture 39:577-582. DOI: 10.1016/j.gaitpost.2013.09.014.

Schwartz MH., Rozumalski A., Trost JP. 2008. The effect of walking speed on the gait of typically developing children. Journal of Biomechanics 41:1639-1650. DOI: 10.1016/j.jbiomech.2008.03.015.

Simonsen EB., Dyhre-Poulsen P., Voigt M., Aagaard P., Fallentin N. 1997. Mechanisms contributing to different joint moments observed during human walking. Scandinavian journal of medicine \& science in sports 7:1-13. DOI: 10.1111/j.1600-0838.1997.tb00110.x.

Stebbins J., Harrington M., Thompson N., Zavatsky A., Theologis T. 2006. Repeatability of a model for measuring multi-segment foot kinematics in children. Gait and Posture. DOI: 10.1016/j.gaitpost.2005.03.002.

Tulchin K., Orendurff M., Adolfsen S., Karol L. 2009. The effects of walking speed on multisegment foot kinematics in adults. Journal of Applied Biomechanics 25:377-386. DOI: 10.1123/jab.25.4.377.

Umberger BR., Martin PE. 2007. Mechanical power and efficiency of level walking with different stride rates. Journal of Experimental Biology 210:3255-3265. DOI: 10.1242/jeb.000950.

White SC., Tucker CA., Brangaccio JA., Lin HY. 1996. Relation of Vertical Ground Reaction Forces to Walking Speed. Gait and Posture 4:206.

Wright CJ., Arnold BL., Coffey TG., Pidcoe PE. 2011. Repeatability of the modified Oxford foot model during gait in healthy adults. Gait and Posture 33:108-112. DOI: 
$541 \quad$ 10.1016/j.gaitpost.2010.10.084.

542 
Figure 1

Oxford foot model marker placement.

The three markers (LD1M, LMMA, and LPCA) were removed after static modeling.

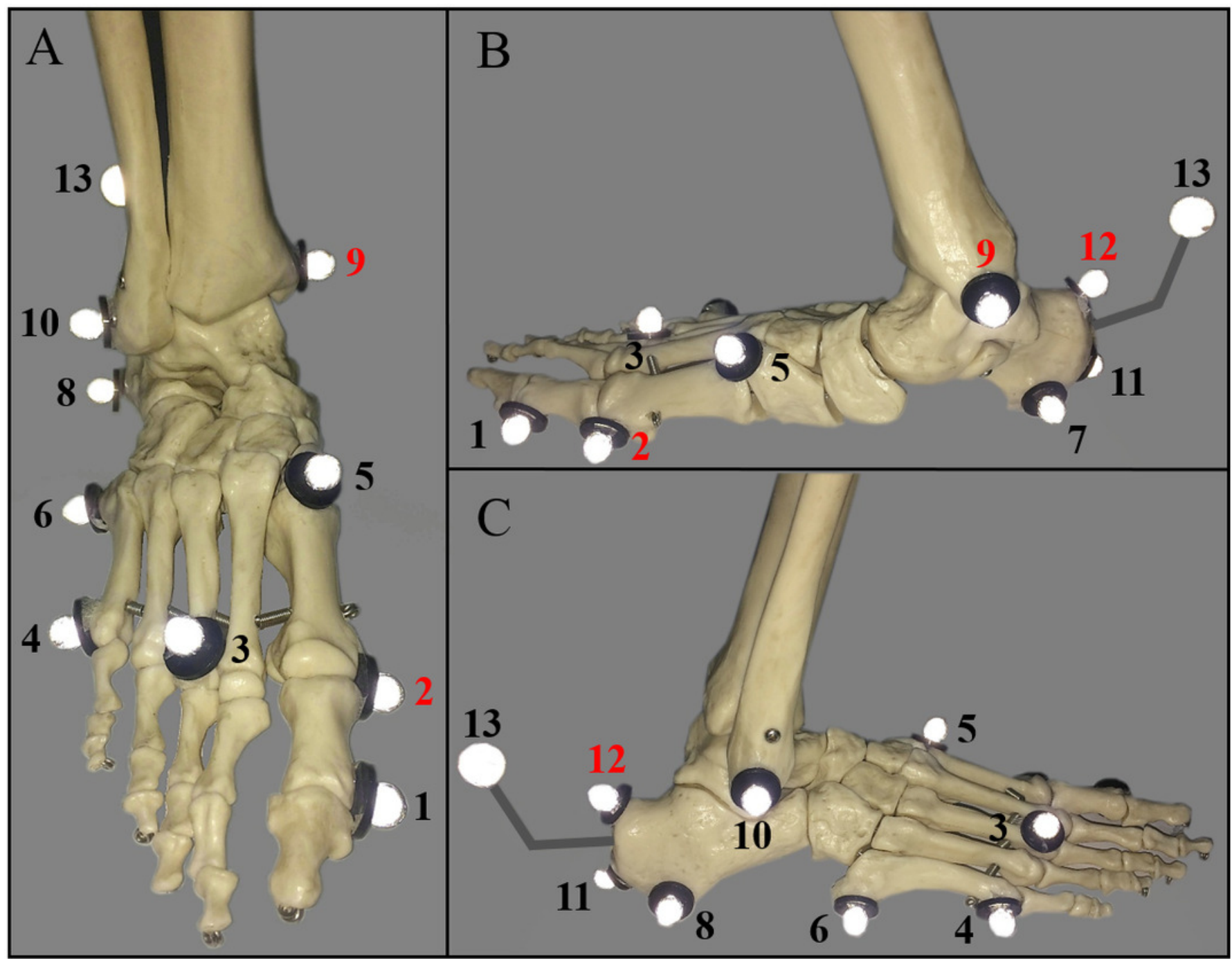

$\begin{array}{lllll}\text { 1: RHLX } & \text { 4: RD5M } & \text { 7: RSTL } & \text { 10: RANK } & \text { 13: RCPG } \\ \text { 2: RD1M } & \text { 5: RP1M } & \text { 8: RLCA } & \text { 11: RHEE } & \\ \text { 3: RTOE } & \text { 6: RP5M } & \text { 9: RMMA } & \text { 12: RPCA } & \end{array}$




\section{Figure 2 (on next page)}

Average foot kinematics $\left(^{\circ}\right)$ in sagittal, frontal and transverse planes during the gait cycle, shaded bands shows mean \pm standard deviation across three walking speeds.

(SP, SP' and SP"' represents the stance phase of FW, NW and SW conditions). 


\section{Table 1 (on next page)}

Kinematic sign conventions used for OFM inter-segment angles 
Table 1. Kinematic sign conventions used for Oxford Foot Model segment angles

\begin{tabular}{llll}
\hline Foot segment angles & Sagittal & Frontal & Transverse \\
\hline Hindfoot/Tibia & Dorsiflexion (DF): + & Inversion (IV): + & Internal rotation (IR): + \\
& Plantarflexion (PF): - & Eversion (EV): - & External rotation (ER): - \\
Forefoot/Hindfoot & Dorsiflexion (DF): + & Supination (SP): + & Adduction (ADD): + \\
& Plantarflexion (PF): - & Pronation (PR): - & Abduction (ABD): - \\
Forefoot/Tibia & Dorsiflexion (DF): + & Supination (SP): + & Adduction (ADD): + \\
& Plantarflexion (PF): - & Pronation (PR): - & Abduction (ABD): - \\
Hallux/Forefoot & Dorsiflexion (DF): + & $\backslash$ & $\backslash$ \\
& Plantarflexion (PF): & $\backslash$ & $\backslash$ \\
\hline
\end{tabular}

2 Note. * Sagittal plane only 


\section{Table 2 (on next page)}

Descriptive statistics of the temporal-spatial parameters (mean \pm SD) during three walking speeds. 
1

2 Table 2. Descriptive statistics of the temporal-spatial parameters (mean \pm SD) during three

3 walking speeds.

\begin{tabular}{|c|c|c|c|}
\hline Variables & SW & NW & FW \\
\hline Walking speed $^{* *}(\mathrm{~m} / \mathrm{s})$ & $1.01 \pm 0.06$ & $1.34 \pm 0.05$ & $1.69 \pm 0.12$ \\
\hline Stride time ${ }^{* *}(\mathrm{~s})$ & $1.11 \pm 0.02$ & $0.96 \pm 0.02$ & $0.79 \pm 0.02$ \\
\hline Stance duration $^{* *}(\mathrm{~s})$ & $0.65 \pm 0.02$ & $0.55 \pm 0.02$ & $0.39 \pm 0.02$ \\
\hline Stance phase* (\% gait cycle) & $57.99 \pm 7.81$ & $56.31 \pm 6.54$ & $48.27 \pm 2.84$ \\
\hline Cadence $^{* *}$ (strides/min) & $108.6 \pm 3.9$ & $126.5 \pm 2.9$ & $154.3 \pm 4.5$ \\
\hline Stride length* ${ }^{*}(\mathrm{~m})$ & $1.31 \pm 0.04$ & $1.37 \pm 0.04$ & $1.42 \pm 0.06$ \\
\hline
\end{tabular}

4 Note. * Statistical Significance $p<0.01, * *$ Statistical Significance $p<0.001$.

5

6 


\section{Table 3 (on next page)}

Foot kinematics with three walking speeds in the stance phase of gait. 
1

2 Table 3. Foot kinematics in the stance phase of gait. Angular rotation of the hindfoot relative

3 to the tibia (HF/TB), the forefoot relative to the $h i n d f o o t(F F / H F)$, the forefoot relative to the

4 tibia (FF/TB) and the hallux relative to the forefoot (HX/FF).

\begin{tabular}{|c|c|c|c|c|c|c|c|}
\hline \multirow{2}{*}{ Variables } & \multicolumn{3}{|c|}{ Walking speed } & \multirow{2}{*}{$\begin{array}{l}\text { ANOVA } \\
p \text { value }\end{array}$} & \multicolumn{3}{|c|}{ Post hoc test ( $p$ value) } \\
\hline & SW & NW & FW & & $\mathrm{SW} / \mathrm{NW}$ & SW/FW & $\mathrm{NW} / \mathrm{FW}$ \\
\hline \multicolumn{8}{|c|}{ Hindfoot/Tibia (HF/TB) } \\
\hline $\mathrm{DF}(\mathrm{IC})$ & $5.7 \pm 1.6$ & $6.0 \pm 2.2$ & $9.7 \pm 1.3$ & $<0.001$ & 0.17 & $<0.001$ & $<0.001$ \\
\hline DF (max) & $19.1 \pm 1.2$ & $17.7 \pm 0.9$ & $29.5 \pm 1.5$ & $<0.001$ & 0.06 & $<0.001$ & $<0.001$ \\
\hline $\mathrm{PF}(\max )$ & $0.1 \pm 3.0$ & $-0.3 \pm 1.6$ & $-0.6 \pm 2.2$ & 0.33 & 0.16 & 0.53 & 0.84 \\
\hline ROM (DF/PF) & $21.0 \pm 1.4$ & $22.3 \pm 1.7$ & $30.1 \pm 2.9$ & $<0.001$ & 0.17 & $<0.001$ & $<0.001$ \\
\hline EV (IC) & $-9.0 \pm 1.6$ & $-10.1 \pm 2.0$ & $-13.8 \pm 1.3$ & $<0.001$ & 0.43 & $<0.001$ & $<0.001$ \\
\hline $\mathrm{EV}(\max )$ & $-22.6 \pm 1.6$ & $-21.2 \pm 2.1$ & $-21.5 \pm 1.2$ & 0.29 & 0.12 & 0.31 & 0.53 \\
\hline IV (max) & $3.2 \pm 1.8$ & $3.9 \pm 0.8$ & $-0.3 \pm 1.5$ & $<0.001$ & 0.31 & $<0.001$ & $<0.001$ \\
\hline ROM (IV/EV) & $25.8 \pm 1.64$ & $25.0 \pm 2.4$ & $21.2 \pm 2.7$ & $<0.001$ & 0.55 & $<0.001$ & $<0.001$ \\
\hline IR (IC) & $2.7 \pm 0.8$ & $2.9 \pm 1.1$ & $2.6 \pm 0.7$ & 0.67 & 0.71 & 0.61 & 0.38 \\
\hline ER (max) & $-2.0 \pm 0.6$ & $-2.3 \pm 0.6$ & $-9.0 \pm 0.7$ & $<0.001$ & 0.70 & $<0.001$ & $<0.001$ \\
\hline IR (max) & $7.2 \pm 1.5$ & $8.4 \pm 0.5$ & $5.6 \pm 0.8$ & 0.59 & 0.28 & 0.39 & 0.72 \\
\hline ROM (IR/ER) & $9.2 \pm 1.3$ & $10.4 \pm 1.1$ & $14.6 \pm 1.1$ & $<0.001$ & 0.11 & $<0.001$ & $<0.001$ \\
\hline \multicolumn{8}{|c|}{ Forefoot/Hindfoot (FF/HF) } \\
\hline DF (IC) & $2.8 \pm 1.7$ & $3.5 \pm 1.0$ & $3.8 \pm 1.8$ & 0.14 & 0.35 & 0.08 & 0.30 \\
\hline $\mathrm{DF}(\max )$ & $9.0 \pm 0.7$ & $9.2 \pm 0.5$ & $12.1 \pm 1.2$ & 0.01 & 0.23 & $<0.001$ & 0.02 \\
\hline $\mathrm{PF}(\max )$ & $-9.4 \pm 1.4$ & $-10.5 \pm 0.9$ & $-5.6 \pm 0.4$ & $<0.001$ & 0.03 & $<0.001$ & $<0.001$ \\
\hline ROM (DF/PF) & $18.3 \pm 1.4$ & $19.8 \pm 0.9$ & $14.2 \pm 1.2$ & $<0.001$ & 0.03 & $<0.001$ & $<0.001$ \\
\hline $\mathrm{SP}(\mathrm{IC})$ & $12.2 \pm 0.5$ & $12.4 \pm 0.4$ & $10.7 \pm 0.7$ & $<0.001$ & 0.53 & $<0.001$ & $<0.001$ \\
\hline $\mathrm{SP}(\min )$ & $6.6 \pm 0.3$ & $6.5 \pm 0.3$ & $5.9 \pm 0.3$ & $<0.001$ & 0.98 & $<0.001$ & $<0.001$ \\
\hline $\mathrm{SP}(\max )$ & $14.6 \pm 0.8$ & $14.5 \pm 0.6$ & $13.8 \pm 0.5$ & 0.03 & 0.86 & 0.02 & 0.03 \\
\hline ROM (SP/PR) & $8.0 \pm 0.8$ & $8.0 \pm 0.5$ & $7.9 \pm 0.6$ & 0.95 & 0.86 & 0.75 & 0.90 \\
\hline ADD (IC) & $4.0 \pm 1.6$ & $3.7 \pm 1.7$ & $-1.3 \pm 0.9$ & $<0.001$ & 0.72 & $<0.001$ & $<0.001$ \\
\hline ABD (max) & $-3.4 \pm 0.9$ & $-2.8 \pm 0.6$ & $-4.7 \pm 0.6$ & $<0.001$ & 0.14 & 0.01 & $<0.001$ \\
\hline
\end{tabular}




$\begin{array}{lccccccc}\text { ABD (TO) } & -1.6 \pm 0.7 & -2.3 \pm 0.6 & -1.6 \pm 0.9 & 0.15 & 0.09 & 0.90 & 0.09 \\ \text { ROM } & 7.4 \pm 1.1 & 6.8 \pm 1.2 & 5.1 \pm 0.5 & <0.001 & 0.20 & <0.001 & 0.02\end{array}$

\section{Forefoot/Tibia (FF/TB)}

$\begin{array}{lccccccc}\text { DF (IC) } & 8.6 \pm 1.1 & 9.0 \pm 1.1 & 14.4 \pm 1.4 & <0.001 & 0.44 & <0.001 & <0.001 \\ \text { DF (max) } & 19.3 \pm 1.3 & 17.8 \pm 1.3 & 33.4 \pm 1.8 & <0.001 & 0.04 & <0.001 & <0.001 \\ \text { PF (max) } & -7.0 \pm 4.0 & -12.7 \pm 2.2 & -4.8 \pm 2.2 & <0.001 & <0.001 & 0.12 & <0.001 \\ \text { ROM (DF/PF) } & 26.3 \pm 3.5 & 30.6 \pm 2.8 & 38.2 \pm 3.2 & <0.001 & 0.01 & <0.001 & <0.001 \\ \text { SP (IC) } & 3.2 \pm 1.5 & 2.2 \pm 1.9 & -3.3 \pm 1.5 & <0.001 & 0.23 & <0.001 & <0.001 \\ \text { PR (max) } & -13.7 \pm 1.9 & -12.2 \pm 2.2 & -12.8 \pm 1.4 & 0.22 & 0.09 & 0.29 & 0.45 \\ \text { SP (max) } & 15.6 \pm 2.9 & 17.5 \pm 1.2 & 13.9 \pm 1.8 & 0.01 & 0.07 & 0.03 & 0.02 \\ \text { ROM (SP/PR) } & 29.3 \pm 2.9 & 29.7 \pm 1.9 & 26.8 \pm 1.8 & 0.12 & 0.73 & 0.15 & 0.09 \\ \text { ADD (IC) } & 6.2 \pm 1.5 & 6.0 \pm 1.9 & 0.4 \pm 1.3 & <0.001 & 0.84 & <0.001 & <0.001 \\ \text { ABD (max) } & -5.6 \pm 1.1 & -4.8 \pm 0.9 & -14.2 \pm 0.9 & <0.001 & 0.11 & <0.001 & <0.001 \\ \text { ADD (TO) } & 7.5 \pm 1.0 & 7.8 \pm 1.0 & 4.1 \pm 1.3 & <0.001 & 0.51 & <0.001 & <0.001 \\ \text { ROM } & 13.1 \pm 1.3 & 12.7 \pm 0.7 & 18.2 \pm 1.8 & <0.001 & 0.55 & <0.001 & <0.001\end{array}$

\section{Hallux/Forefoot (HX/FF)}

$\begin{array}{lccccccc}\text { DF (IC) } & 27.3 \pm 3.6 & 28.7 \pm 3.0 & 25.5 \pm 4.0 & 0.18 & 0.39 & 0.31 & 0.07 \\ \text { DF (min) } & 7.8 \pm 1.7 & 7.5 \pm 1.2 & 11.3 \pm 0.7 & <0.001 & 0.98 & <0.001 & <0.001 \\ \text { DF (max) } & 46.1 \pm 3.5 & 45.4 \pm 2.7 & 40.0 \pm 2.3 & <0.001 & 0.56 & <0.001 & <0.001 \\ \text { ROM (DF/PF) } & 37.5 \pm 3.2 & 38.1 \pm 2.5 & 26.6 \pm 2.5 & <0.001 & 0.51 & <0.001 & <0.001\end{array}$

5 Note. $\mathrm{SW}=$ slow walking, $\mathrm{NW}=$ normal walking, $\mathrm{FW}=$ fast walking, $\mathrm{DF}=$ Dorsiflexion,

$6 \mathrm{PF}=$ Plantar-flexion, $\mathrm{SP}=$ Supination, $\mathrm{PR}=$ Pronation, $\mathrm{ADD}=$ Adduction, $\mathrm{ABD}=\mathrm{Abduction}$, $7 \mathrm{IV}=$ Inversion, $\mathrm{EV}=$ Eversion, $\mathrm{IR}=\mathrm{Int}$. rotation, $\mathrm{ER}=$ Ext. rotation, ROM=Range of motion, $8 \mathrm{IC}=$ initial contact, $\mathrm{TO}=$ toe off, $\max =$ maximum, $\min =$ minimum.

9 


\section{Table 4(on next page)}

Comparison of GRFs and peak joint moments at three walking speed. 
1

Table 4. Comparison of GRFs and peak joint moments at three walking speed.

\begin{tabular}{|c|c|c|c|c|c|c|c|}
\hline \multirow{2}{*}{ Variables } & \multicolumn{3}{|c|}{ Walking speed } & \multirow{2}{*}{$\begin{array}{l}\text { ANOVA } \\
p \text { value }\end{array}$} & \multicolumn{3}{|c|}{ Post hoc test ( $p$ value) } \\
\hline & SW & NW & FW & & SW/NW & SW/FW & $\mathrm{NW} / \mathrm{FW}$ \\
\hline \multicolumn{8}{|l|}{ Ground reaction forces } \\
\hline Peak braking (BW) & $0.21 \pm 0.03$ & $0.22 \pm 0.02$ & $0.26 \pm 0.03$ & 0.01 & 0.39 & 0.01 & 0.03 \\
\hline Peak propulsive (BW) & $0.23 \pm 0.01$ & $0.24 \pm 0.01$ & $0.28 \pm 0.03$ & 0.01 & 0.54 & 0.01 & $<0.001$ \\
\hline Peak vertical (BW) & $1.36 \pm 0.07$ & $1.42 \pm 0.04$ & $1.71 \pm 0.05$ & $<0.001$ & 0.28 & 0.02 & $<0.001$ \\
\hline VALR (BW/s) & $9.99 \pm 1.03$ & $12.04 \pm 0.97$ & $18.56 \pm 1.44$ & $<0.001$ & 0.03 & $<0.001$ & $<0.001$ \\
\hline \multicolumn{8}{|c|}{ Joint moments (peak values) $(\mathrm{Nm} / \mathrm{kg})$} \\
\hline Knee flexion & $0.25 \pm 0.06$ & $0.31 \pm 0.09$ & $0.45 \pm 0.11$ & $<0.001$ & 0.02 & $<0.001$ & $<0.001$ \\
\hline Knee extension & $0.62 \pm 0.13$ & $0.65 \pm 0.14$ & $0.76 \pm 0.13$ & $<0.001$ & 0.26 & $<0.001$ & $<0.001$ \\
\hline Knee adduction & $0.08 \pm 0.05$ & $0.08 \pm 0.07$ & $0.12 \pm 0.06$ & 0.01 & 0.62 & $<0.001$ & $<0.001$ \\
\hline Ankle dorsiflexion & $0.21 \pm 0.03$ & $0.15 \pm 0.02$ & $0.18 \pm 0.03$ & 0.01 & 0.11 & $<0.001$ & 0.04 \\
\hline Ankle plantarflexion & $0.37 \pm 0.07$ & $0.41 \pm 0.08$ & $0.51 \pm 0.13$ & $<0.001$ & 0.16 & $<0.001$ & $<0.001$ \\
\hline Ankle eversion & $0.11 \pm 0.01$ & $0.11 \pm 0.02$ & $0.14 \pm 0.02$ & 0.01 & 0.35 & 0.02 & $<0.001$ \\
\hline
\end{tabular}

3 Note. $\mathrm{BW}=$ body weight, VALR=vertical average loading rate.

4 